\title{
Image decomposition and uncertainty quantification for the assessment of manufacturing tolerances in stress analysis
}

\author{
Gabriele Marcuccio, Elvio Bonisoli and Stefano Tornincasa
}

Politecnico di Torino, Department of Management and Production Engineering Corso Duca degli Abruzzi, 24 - 10129 Torino, Italy

\section{John E. Mottershead and Edoardo Patelli}

Institute for Risk and Uncertainty, University of Liverpool

The Quadrangle, Brownlow Hill, Liverpool L69 3GH, United Kingdom

\section{Weizhuo Wang}

School of Engineering, Manchester Metropolitan University, Manchester M1 5GD, United Kingdom

e-mail: gabriele.marcuccio@polito.it

Keywords: Interference fit, shape descriptor, polynomial chaos expansion, global sensitivity analysis, dimensional tolerances.

\begin{abstract}
This paper presents a methodology for the treatment of uncertainty in nonlinear, interference-fit, stress analysis problems arising from manufacturing tolerances. Image decomposition is applied to the uncertain stress field to produce a small number of shape descriptors that allow for variability in the location of high stress points when geometric parameters (dimensions) are changed within tolerance ranges. A meta-model, in this case based on the polynomial chaos expansion (PCE), is trained using a full finite element model to provide a mapping from input geometric parameters to output shape descriptors. Global sensitivity analysis using Sobol' indices provides a design tool that enables the influence of each input parameter on the observed variances of the outputs to be quantified. The methodology is illustrated by a simplified practical design problem in the manufacture of automotive wheels.
\end{abstract}




\section{Introduction}

The design process is expected to deliver continuing reductions in manufacturing and material costs without compromising the performance of assemblies of engineering components. Modern light-weight parts, achieved by the use to thinner gauge raw materials, are generally more flexible than the heavier components that they replaced. Manufacturing tolerances can then become critical to the understanding of stress distributions, requiring a stochastic approach to ensure satisfactory performance. Commercial systems, such as eMTolMate (TecnoMatix) or CATIA 3D FTA and CETOL $6 \sigma$ (Sigmetrix), are available for the setting and assessment of tolerances, but do not take account of the flexibility of components affected by deformation during the assembly/manufacturing process. These systems are restricted to nominal rigid-body component assemblies.

This paper proposes a stochastic design methodology for the assessment of stress distributions in press-fitted (interference fit) components. Finite element (FE) stress fields are represented in the form shape descriptors obtained by image decomposition in known high-stress regions. Image decomposition was developed for the representation of full-field measurements, displacements or strains, obtained typically by Digital Image Correlation (DIC), where it has been demonstrated to reproduce the information contained in $10^{4}-10^{5}$ measurement points by a few tens of shape descriptors without any significant loss of accuracy. The shape descriptors are properties of the full-field data, rather than a collection of point-wise discrete values. This is an important aspect of the methodology because it means that high-stress points are always captured by the shape descriptors, even if the location of the high stress points should change as a result of dimensional variability (within manufacturing tolerances).

The high computational cost of multiple FE stress calculations is reduced to acceptable levels on modern workstations by the application of meta-models, the purpose of which is to represent the complicated relationship between variable FE parameters (the input) and the shape descriptors (the output) using a much simpler 'surrogate' input-output relationship. Efficient training and subsequent use of a 
meta-model requires sampling of the input. Random sampling is known to be inefficient and inferior to Latin Hypercube Sampling (LHS) which from sample to sample exhibits less variability in estimating the sample mean and the cumulative distribution function (CDF). Many different meta-models are available including, polynomial regression, neural networks, the polynomial chaos expansion (PCE) and Gaussian process emulation (Kriging). The trained emulator may also be used to provide global sensitivity estimates from the fractional contribution of each input to the variance of the output. Global Sensitivity Analysis (GSA) may be carried out very efficiently when the output is expressed as a truncated PCE and provides a basis for ranking the importance of the various inputs.

The paper uses the simplified example of an automotive wheel, consisting of two components, an outer rim with a press fitted (interference fit) internal disc, to explain the working of the design methodology, including the use of image analysis, a PCE meta-model and GSA to assess the effect of manufacturing tolerances on the FE stress field expressed in terms of shape descriptors. The particular wheel considered is manufactured by the Italian company MW S.p.A. FE models of the rim and disc are shown in Figure 1 and the dimensions of the two parts are of course variable with manufacturing tolerances. It can be appreciated that the tolerance on the rim diameter will be considerably greater than the disc because of the high expense of tight tolerances on such a complicated pressed, rolled and welded component.
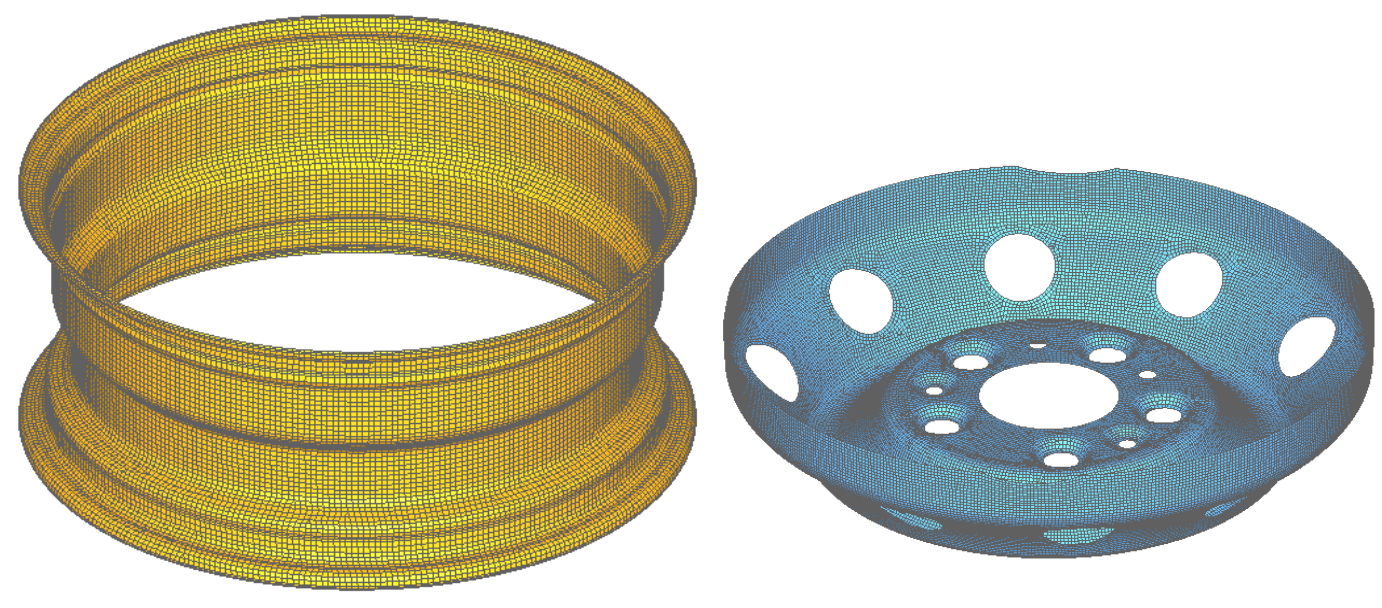

Figure 1: FE Models of the Rim and Disc 


\section{Methodology}

The design methodology is explained graphically in Figure 2. Manufacturing tolerances form part of the design specification, which in turn affects the stress distribution in the assembled system of components. The meta-model, determined by training, becomes a surrogate for the full FE model, enabling the effect of manufacturing tolerances on the stress field to be determined efficiently using image decomposition. Global sensitivity analysis enables an assessment to be made on the contribution of each input to the variability of each output. We assume the inputs to be uniformly distributed within manufacturing tolerance bounds and determine the contribution of each to the highest bound on each of the outputs.

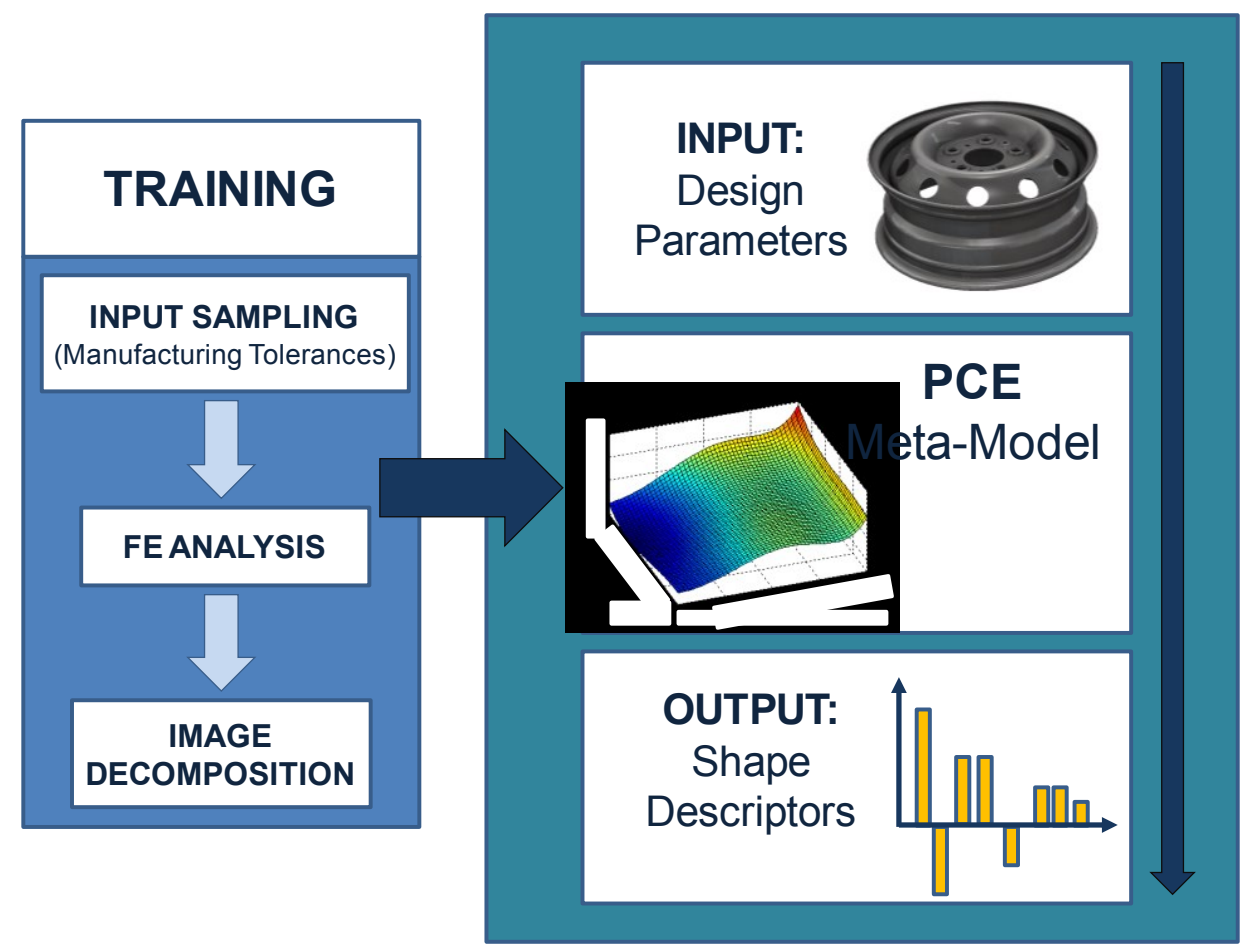

Figure 2: Design Methodology

\section{Illustrative Example: Automotive Wheel}

The wheel assembly is composed of a rim and a disc, both of which are steel pressings. The disc is made of a material equivalent to DP600 (dual phase steel), 
whereas the rim is FEP11. The assembly is press fitted (interference fit) and welded along the boundary between the disc and the rim. Regions of high stress are known to exist in five specific locations shown in Figure 3. These are: 1) close to the rim well where the material thickness is reduced due to press forming of the rim profile; 2 ) in the weld zone; 3) close to the vent holes where cracks can nucleate and propagate and where the maximum circumferential stresses are found; 4) at the hat radius where there are high bending stresses; and 5) in the bolt contact area where disc failure is caused by fretting. For purposes of illustration, we consider only the stress field in the region of the vent hole though the methodology may be readily extended to include the other four critical regions simultaneously. Wang and Zhang ${ }^{1}$ show stress plots with high von Mises stresses in the region of the vent hole, albeit under different loading, and although the vent holes are remote from the interference-fit region, stresses around them are known to be affected by the degree of interference ${ }^{1}$.

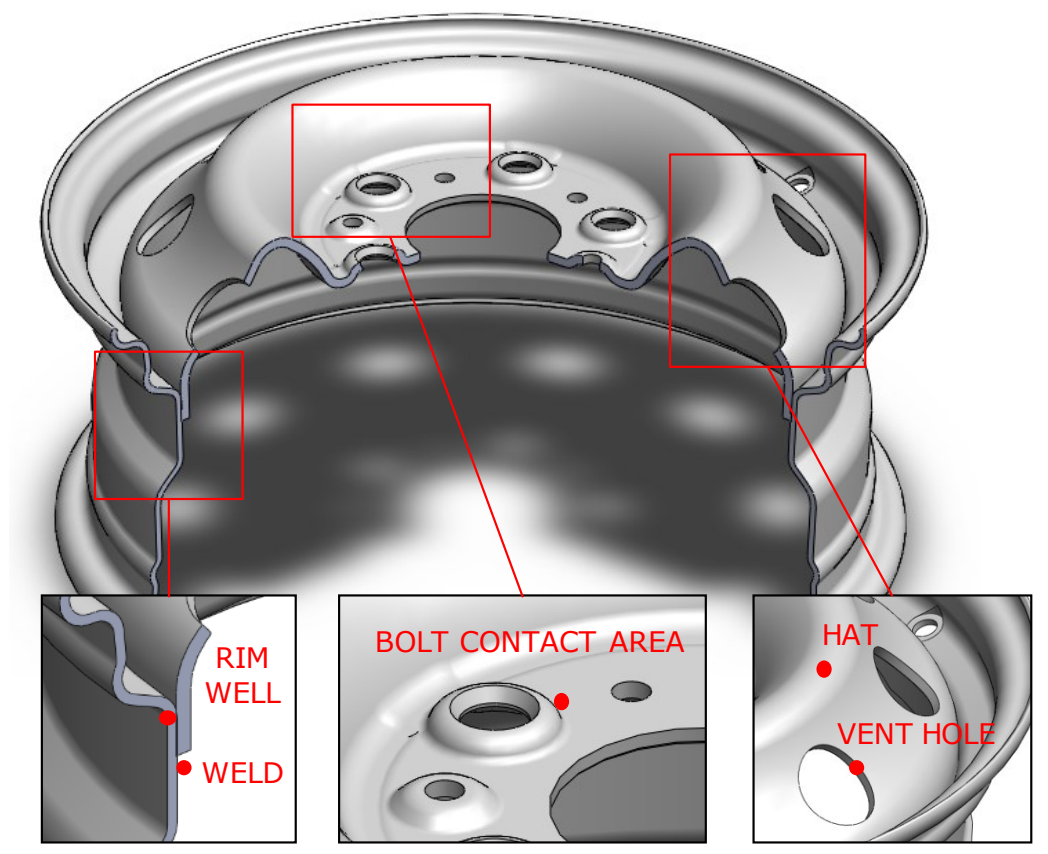

Figure 3: Automotive wheel comprising disc and rim marked with regions of high stress

\footnotetext{
${ }^{1}$ An example of the distribution of stresses in different critic areas of a passenger car wheel can be found in Grubisic and Fischer ${ }^{2}$.
} 
Grubisic and Fisher pointed out that considerable improvements in wheel design could be achieved by an improved understanding of the process of the interferencefit and its effect on the stress fields in the mating parts ${ }^{2}$.

The following material properties were used in the construction of a FE model: Young's modulus $E=210 \mathrm{GPa}$, Poisson ratio $v=0.3$, density $\rho=7900 \mathrm{~kg} / \mathrm{m}^{3}$. In FE analysis all the degrees of freedom of the outer flange rim were constrained, and symmetry boundary conditions were applied to a $1 / 4$ segment. This is a simplification of the industrial problem which includes uncertainly due to eccentricity and deviation from circularity, both of which are neglected here - as is the weld between the disc and rim - for the purpose of illustrating the methodology. The FE simulation was carried out using the Abaqus Standard/Explicit code with shell elements (CQUAD4 and CTRIA3) of $2 \mathrm{~mm}$ nominal dimension. Elasto-plastic material with isotropic hardening was used in the analysis of the fitting process and spring-back phases shown in Figure 4. The contact analysis was carried out using a penalty function to prevent the mutual penetration of the disc and rim meshes during the press-fitted assembly. The surface-to-surface constraint allows finite mutual sliding over 'hard' surfaces with pressure overclosure and a Coulomb friction coefficient of 0.4 .

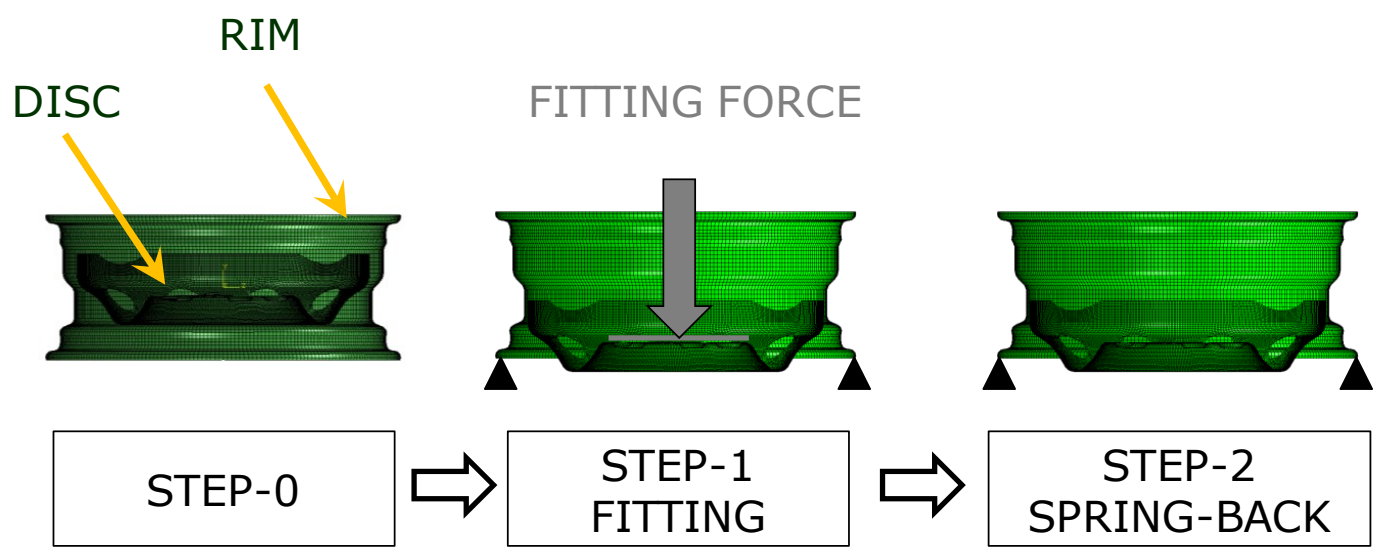

Figure 4: FE application of interference fit

\section{Image Decomposition}


Modern image analysis techniques have been applied to full-field measurement of displacement and strain by Wang et al. ${ }^{3,4}$. These methods are designed for use with full-field data such as those obtained by Digital Image Correlation (DIC) but can equally be used with finite element predictions. They have the advantage that a displacement, strain or stress field can be condensed to a small number of image descriptors (sometimes called shape features or moment descriptors).

Two-dimensional moment invariants were initially introduced to recognise plane patterns, to process visual information and to efficiently recapture all the image features in a reduced and compact sequence of real numbers ${ }^{5,6}$. The full field data $w(\mathbf{x})$ may be expressed as a linear combination of orthonormal kernel functions as

$w(\mathbf{x})=\sum_{r=1}^{\infty} \alpha_{r} g_{r}(\mathbf{x})$

where $\{\alpha\}_{r=1}^{\infty}$ are the shape descriptors, $\{g\}_{r=1}^{\infty}$ are the kernels, and $\mathbf{x}$ denotes the spatial domain. For regular domains, either rectangular or circular, the kernels generally take the form of orthogonal polynomials, Legendre or Tchebyshev (continuous or discrete polynomials respectively on rectangular domains), or Zernike (continuous on circular domains). In the case of irregular domains, typical of the surfaces of engineering components, special procedures are required to adapt the classical basis functions. The Adaptive Geometric Moment Descriptor ${ }^{7}$ (AGMD) was described by Wang and Mottershead. In that case two-dimensional monomials are used to construct a set of orthonormal kernel functions, which may be expressed as,

$g_{r}(\mathbf{x})=g_{p, q}(x, \mathrm{y})=g_{r}(x, \mathrm{y})=G S O^{(\Omega)}\left(x^{p} y^{q}\right)$

where $p, q \in\{0,1, \ldots$, are monomial orders, GSO denotes Gram-Schmidt orthonormalisation $^{8}$ and $x, y$ are coordinates defined on the continuous spatial domain $\Omega$ . The shape features (or AGMDs) are given by the projection of the image onto each kernel function using the unweighted inner product as $\alpha_{r}=\int_{\Omega} w(\mathbf{x}) g_{r}(\mathbf{x}) d \mathbf{x}$. FE surface stress patterns or full-field measurements are usually defined on discrete 
meshes e.g. on a triangularised mesh. The shape features, $\alpha_{r}$, may then be evaluated by the discrete inner product as

$\alpha_{r}=\sum_{k=1}^{\text {nodes }} w\left(x_{k}, y_{k}\right) g_{r}\left(x_{k}, y_{k}\right) \Delta_{k} ; \quad r=1,2, \ldots$

where $x_{k}, y_{k}$ are the coordinates of the $k^{\text {th }}$ node, $\Delta_{k}$ is an area associated with each node that arises from the numerical integration and $m$ denotes the number of shape descriptors (outputs). The Voronoi cell ${ }^{9}$ may be applied to approximate the integration area surrounding each node and is adopted in this paper. Wang and Mottershead $^{7}$ describe a process of surface parameterisation (conformal mapping) that allows curved surfaces, such as the surfaces of the disc and hub, to be mapped to $2 \mathrm{D}$ planar surfaces, thereby permitting the procedure described above to be applied.

It is unnecessary to carry out a complete full-field reconstruction of the stress field in the wheel, because high stresses occur in known regions. Also reconstruction over a partial stress field can be achieved using much fewer shape descriptors, as in Figure 5. The reconstruction of the stress field around the vent hole was achieved using 20 AGMDs and it is seen that its features are captured to very good accuracy the error is shown in Figure 5(c). The eight most significant AGMDs are seen in Figure 6 to be $\left(\mathrm{AGMD}_{1}, \mathrm{AGMD}_{2}, \mathrm{AGMD}_{3}, \mathrm{AGMD}_{5}, \mathrm{AGMD}_{4}, \mathrm{AGMD}_{8}, \mathrm{AGMD}_{16}\right.$, AGMD $_{9}$ ) and the Pearson correlation coefficient ${ }^{10}$ (PCC) in Figure 7 shows a $95 \%$ correlation when using 8 AGMDs. 

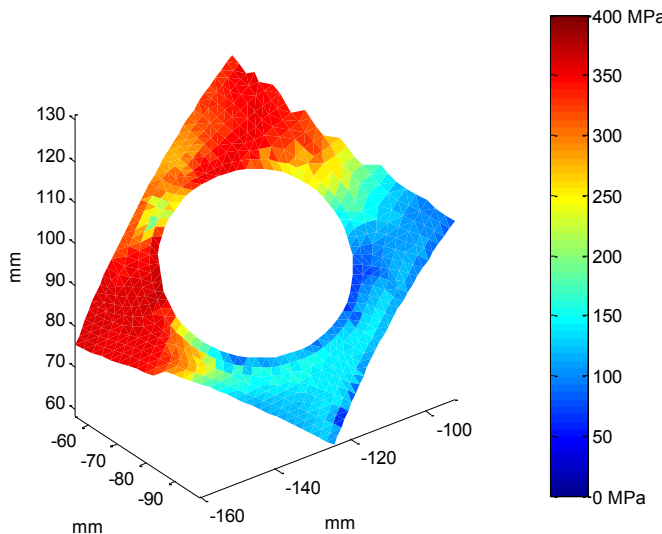

(a)

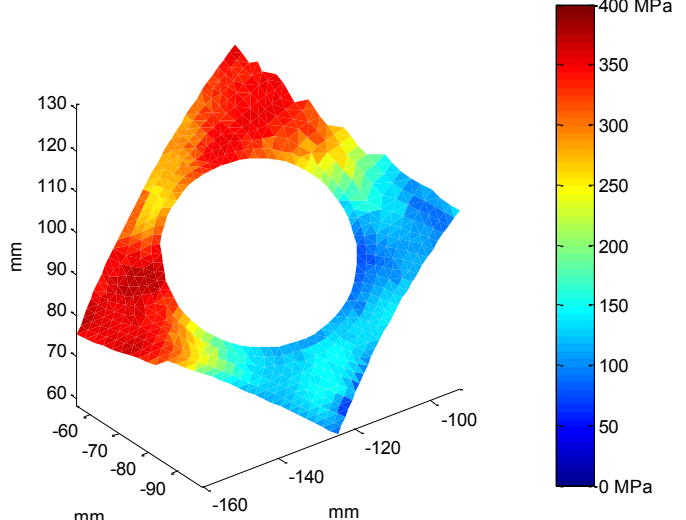

(b)

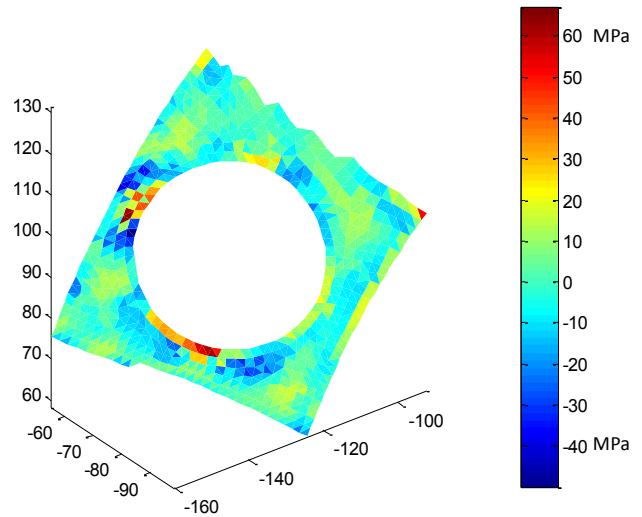

(c)

Figure 5: von Mises stress around a disc vent hole: (a) FE result, (b) reconstructed image using 20 AGMDs, (c) error 


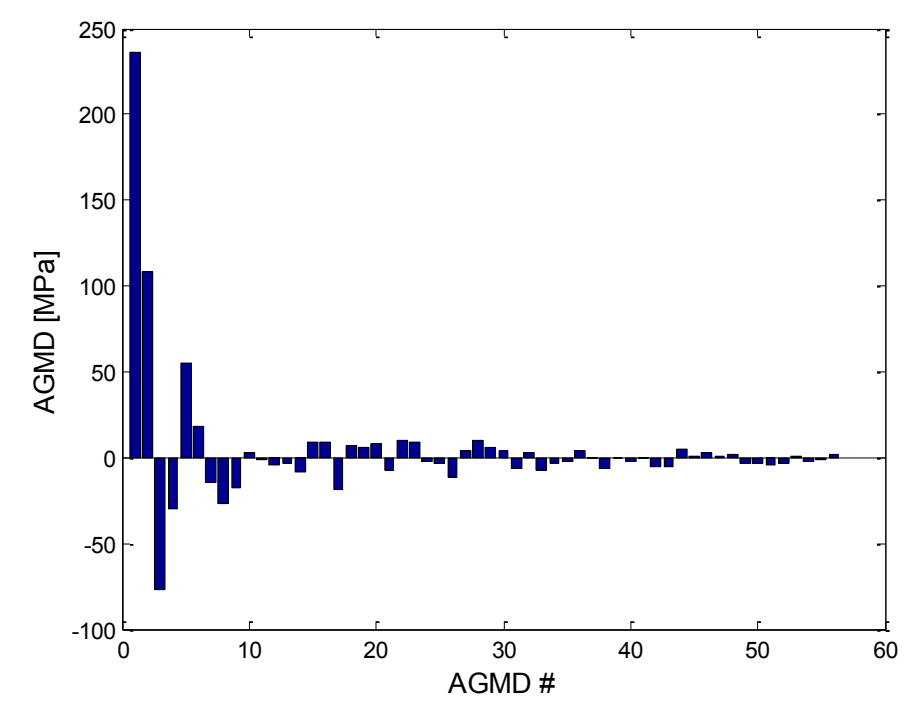

Figure 6: Spectrum of AGMDs showing most significant contributions to the reconstructed stress field.

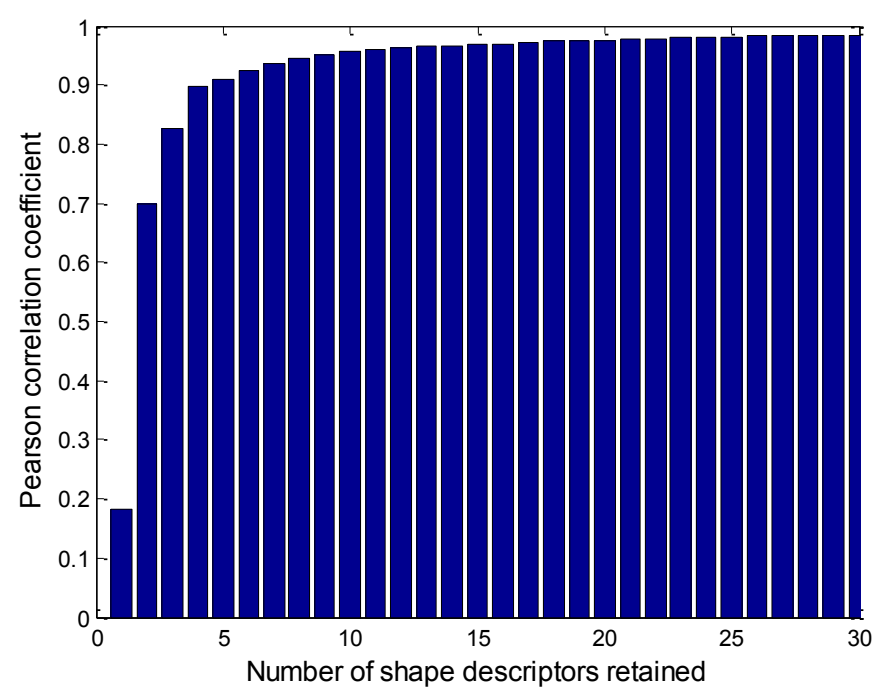

Figure 7: Pearson correlation coefficient between reconstructed full field and original full field

Whereas, for example, the greatest stress might occur in different locations around a vent hole with variability in the process of applying the interference fit, the image descriptors will always be able to capture the resulting variability in the stress field, including the maximum stress independently of its precise location. Moreover different combinations of geometrical and material parameters can be numerically 
tested to provide guidelines for the design and manufacture of engineering components, such as interference-fitted wheels.

\section{Inputs and sampling plans}

The inputs to the stochastic analysis are the design parameters, variable within manufacturing tolerances, that contribute to the interference between the rim and disc. These are the diameters of the rim and disc, $\phi_{r}$ and $\phi_{d}$, and the thicknesses of the plate materials from which the two parts are manufactured, $t_{r}$ and $t_{d}$, shown in Figure 8 .

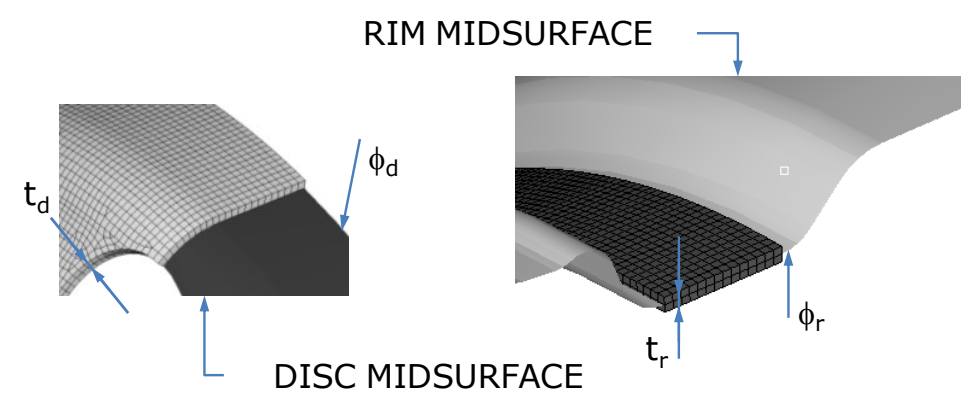

Figure 8: Design parameters (inputs)

Efficient maximin $^{11}$ Latin Hypercube sampling (LHS) was adopted to explore the input space while satisfying the uniformity criterion of LHS and yielding randomized plans which maximise the minimum distance between samples. This approach is designed to minimise the size of the sample to the least possible without loss of reliability of solutions.

Firstly, five different sampling plans $\left\{\mathrm{LHS}_{k}\right\}_{k=1}^{5}$ each of $n$ points uniformly distributed on the four inputs $\left(\left\{\xi_{i}\right\}_{i=1}^{4}=\phi_{d}, \phi_{r}, t_{d}, t_{r}\right)$ were built and analysed. The effect of their variation, in a defined range between upper and lower bounds, on the output response was studied. In the first plan (LHS 1 ) all four inputs were sampled and in subsequent plans $\left(\mathrm{LHS}_{2}, \mathrm{LHS}_{3}, \mathrm{LHS}_{4}, \mathrm{LHS}\right.$ ) only one input was sampled 
while each of the remaining ones remained fixed at a reference value $\left(R_{v}\right)$ as indicated in Table 1.

\section{Press fitting of the rim and disc}

Numerical results were compared to measurements taken during experimental fitting of a disc and rim pair taken from a production batch. The components satisfied the European Tyres and Rim Technical Organization (ETRTO) profile requirements and the applied force was measured and recorded during the press-fit by means of a load cell and data acquisition system. The resulting measurement, taken under conditions of constant (low) press speed, is seen to be highly nonlinear as shown in Figure 9. The oscillations after the first ramp-up phase are due to axial adjustment of the disk with respect to the rim. The experimental results are compared with 200 numerical analyses using the full FE model and sampling plan LHS $_{1}$. The numerical median is seen to be close to experimental curve, which lies consistently within the band delimited by the $25^{\text {th }}$ and $75^{\text {th }}$ percentiles. One possible reason for the observed discrepancy is the assumption of a friction coefficient of 0.4 in the numerical simulation, whereas in reality there is a process of alignment between the rim and disc that takes place within the press and involves a process of stick-slip not represented in the model. However, the general form of the two curves is considered to be sufficiently similar for the purposes of the uncertainty quantification (UQ) carried out in what follows. The randomised inputs (diameters and shell thicknesses) are of course readily adjusted in FE simulation, but are extremely difficult to control in the manufactured product because of practical difficulties such as unavoidable variation in rolled plate thicknesses and wear on press tools.

Table 1 - Sampling plans

\begin{tabular}{lccc}
\hline \multirow{2}{*}{ Input } & \multicolumn{3}{c}{ LHS $1(\mathrm{n}=200)$} \\
\cline { 2 - 4 } & $\begin{array}{c}\text { Lower } \\
\text { bound }\end{array}$ & $\begin{array}{c}\text { Upper } \\
\text { bound }\end{array}$ & Range \\
\hline Disc diameter $\phi_{d}[\mathrm{~mm}]$ & 359.8 & 360 & 0.2 \\
Rim diameter $\phi_{r}[\mathrm{~mm}]$ & 366 & 367 & 1 \\
Disc thickness $t_{d}[\mathrm{~mm}]$ & 4.56 & 4.80 & 0.24 \\
Rim thickness $t_{r}[\mathrm{~mm}]$ & 3.04 & 3.20 & 0.16 \\
\hline
\end{tabular}




\begin{tabular}{|c|c|c|c|c|c|c|c|c|}
\hline \multirow[b]{2}{*}{ Input } & \multicolumn{4}{|c|}{$\mathbf{L H S}_{\mathbf{2}}(\mathrm{n}=40)$} & \multicolumn{4}{|c|}{$\mathbf{L H S}_{3}(\mathrm{n}=40)$} \\
\hline & $\mathrm{Lb}$ & & $\mathrm{Ub}$ & $\mathrm{R}_{\mathrm{V}}$ & $\mathrm{Lb}$ & & $\mathrm{Ub}$ & $\mathrm{R}_{\mathrm{V}}$ \\
\hline$\phi_{d}[\mathrm{~mm}]$ & 359.8 & I & 360 & 359.8 & 359.8 & 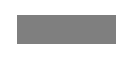 & 360 & - \\
\hline$\phi_{r}[\mathrm{~mm}]$ & 366 & & 367 & - & 366 & I & 367 & 366.6 \\
\hline$t_{d}[\mathrm{~mm}]$ & 4.56 & I & 4.8 & 4.8 & 4.56 & I & 4.8 & 4.8 \\
\hline$t_{r}[\mathrm{~mm}]$ & 3.04 & I & 3.2 & 3.2 & 3.04 & I & 3.2 & 3.2 \\
\hline \multirow[b]{2}{*}{ Input } & \multicolumn{4}{|c|}{$\mathbf{L H S}_{4}(\mathrm{n}=40)$} & \multicolumn{4}{|c|}{$\mathbf{L H S}_{5}(\mathrm{n}=40)$} \\
\hline & $\mathrm{Lb}$ & & $\mathrm{Ub}$ & $\mathrm{R}_{\mathrm{V}}$ & $\mathrm{Lb}$ & & $\mathrm{Ub}$ & $\mathrm{R}_{\mathrm{V}}$ \\
\hline$\phi_{d}[\mathrm{~mm}]$ & 359.8 & I & 360 & 359.8 & 359.8 & I & 360 & 359.8 \\
\hline$\phi_{r}[\mathrm{~mm}]$ & 366 & I & 367 & 366.6 & 366 & I & 367 & 366.6 \\
\hline$t_{d}[\mathrm{~mm}]$ & 4.56 & I & 4.80 & 4.8 & 4.56 & 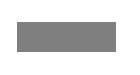 & 4.8 & - \\
\hline$t_{r}[\mathrm{~mm}]$ & 3.04 & 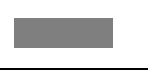 & 3.20 & - & 3.04 & I & 3.2 & 3.2 \\
\hline
\end{tabular}

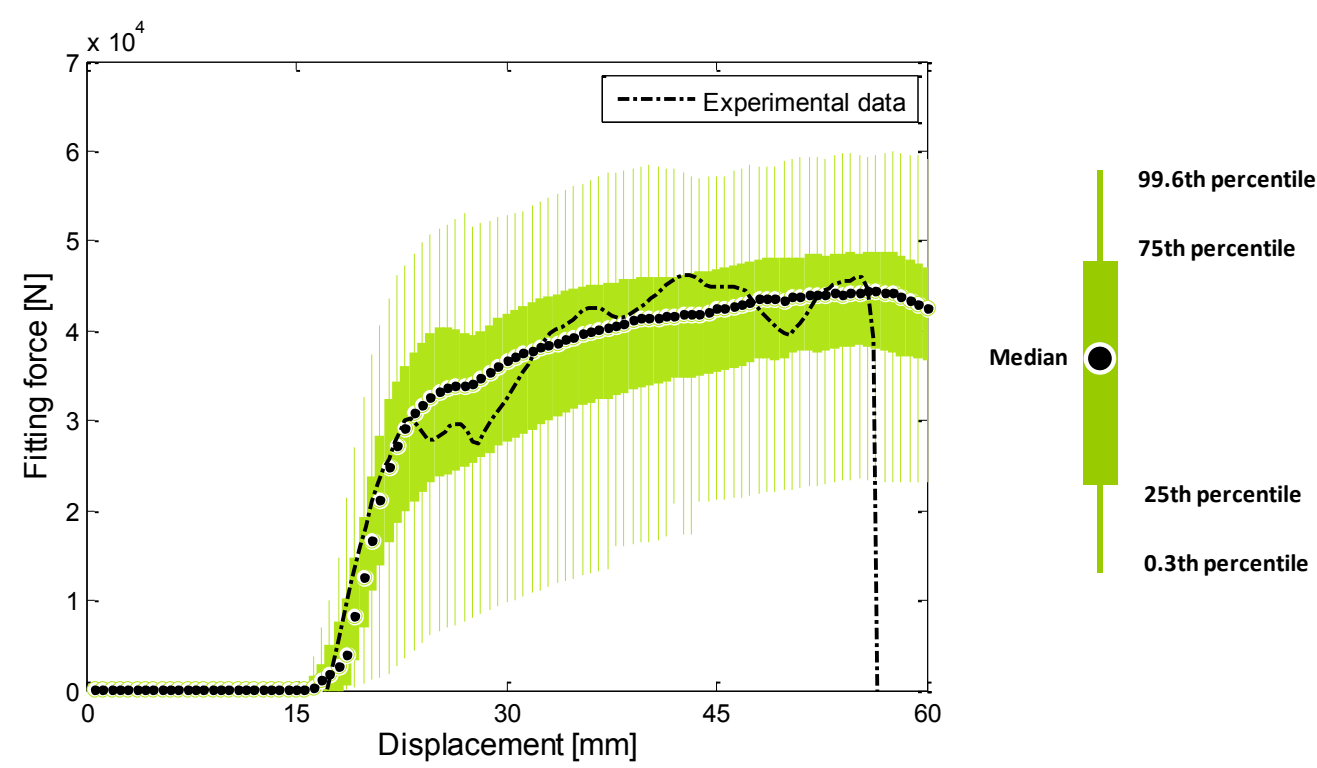

Figure 9: Fitting force trend: experimental data vs. stochastic numerical simulation

\section{Meta-model and uncertainty propagation}

The meta-model used in this research is a multivariate polynomial chaos expansion (PCE). It expresses the AGMD, $\left\{\alpha_{r}(\xi)\right\}_{r=1}^{m}$, as a random function of the inputs. The $\ell$ dimension PCE truncated at the $j^{\text {th }}$ order may be expressed as, 


$$
\begin{gathered}
\alpha_{r}\left(\xi_{i_{j}}\right)=a_{0} \Gamma_{0}+\sum_{i_{1}=1}^{\ell} a_{i_{1}} \Gamma_{1}\left(\xi_{i_{1}}\right)+\sum_{i_{1}=1}^{\ell} \sum_{i_{2}=1} a_{i_{1}, i_{2}} \Gamma_{2}\left(\xi_{i_{1}}, \xi_{i_{2}}\right) \\
+\sum_{i_{1}=1}^{\ell} \sum_{i_{2}=1} \sum_{i_{3}=1} a_{i_{1}, i_{2}, i_{3}} \Gamma_{3}\left(\xi_{i_{1}}, \xi_{i_{2}}, \xi_{i_{3}}\right)+\sum_{i_{1}=1}^{\ell} \sum_{i_{2}=1} \ldots \frac{-}{i_{j}=1}, \ldots, \ldots, \ldots, \ldots, \\
r=1,2, \ldots
\end{gathered}
$$

where $\Gamma_{j}\left(\xi_{i_{1}}, \ldots\right.$, , , are multivariate orthogonal Legendre polynomials in the case of a uniform distribution of inputs $\xi_{i_{j}}$. The process of constructing the polynomials $\Gamma_{j}(\bullet)$ is described by Ghanem and Spanos ${ }^{12}$ so that every polynomial of every order is orthogonal to every other polynomial. The zero ${ }^{\text {th }}$ order polynomial is a constant and may be set to unity. The coefficients $a_{0}, a_{i_{1}}, \ldots, a_{i_{1}, i_{2}, \ldots}$, are assembled in a vector $\mathbf{a}=\left(\begin{array}{llll}a_{0} & a_{1} & \cdots\end{array}, \begin{array}{l}, T \\ ,\end{array}\right.$ and equation (4) may then be recast in the form,

$\boldsymbol{\Psi}_{n \times p} \mathbf{a}_{p \times 1}=\mathbf{h}_{n \times 1} ; \quad n>p=\left(\begin{array}{l}\ell \\ j\end{array}\right)=\frac{\ell}{r ! j !}$

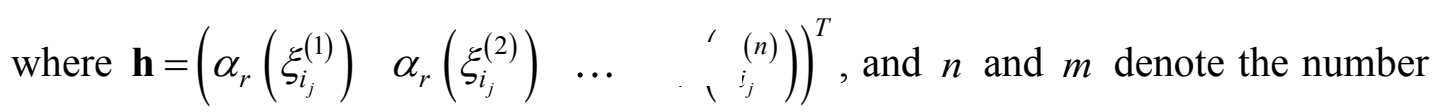
of samples and number of outputs respectively ${ }^{2}$. The coefficients may then be determined using the usual least-squares approximation,

$$
\mathbf{a}=\left(\boldsymbol{\Psi}^{T} \boldsymbol{\Psi}\right)^{-1} \boldsymbol{\Psi}^{T} \mathbf{h}
$$

${ }^{2}$ For clarity, the two-dimensional (two inputs) expansion of equation (4) truncated at the third order may be written as,

$$
\begin{aligned}
& \alpha_{r}=a_{0} \Gamma_{0}+a_{1} \Gamma_{1}\left(\xi_{1}\right)+a_{2} \Gamma_{1}\left(\xi_{2}\right)+a_{11} \Gamma_{2}\left(\xi_{1}, \xi_{1}\right)+a_{12} \Gamma_{2}\left(\xi_{2}, \xi_{1}\right)+a_{22} \Gamma_{2}\left(\xi_{2}, \xi_{2}\right) \\
& +a_{111} \Gamma_{3}\left(\xi_{1}, \xi_{1}, \xi_{1}\right)+a_{211} \Gamma_{3}\left(\xi_{2}, \xi_{1}, \xi_{1}\right)+a_{221} \Gamma_{3}\left(\xi_{2}, \xi_{2}, \xi_{1}\right)+a_{222} \Gamma_{3}\left(\xi_{2}, \xi_{2}, \xi_{2}\right)
\end{aligned}
$$

to be re-cast in the form of equation (5) as,

$$
\alpha_{r}=a_{0} \psi_{0}+a_{1} \psi_{1}+a_{2} \psi_{2}+a_{3} \psi_{3}+a_{4} \psi_{4}+a_{5} \psi_{5}+a_{6} \psi_{6}+a_{7} \psi_{7}+a_{8} \psi_{8}+a_{9} \psi_{9}
$$


The predictivity of the $p^{\text {th }}$ order PC approximation of the model response, the AGMDs, obtained from equations (4) and (6) may be compared to the original FE prediction. The training error $\varepsilon_{\text {training }}$ and the determination coefficient $R^{2}$ are defined for each output as ${ }^{13}$,

$$
\begin{aligned}
& \varepsilon_{r \text { (training })}=\frac{1}{n} \sum_{k=1}^{n}\left(\alpha_{r}\left(\xi^{(k)}\right)-\alpha_{r}^{P C E}\left(\xi^{(k)}\right)\right)^{2} ; \quad r=1,2, \ldots \\
& R_{r}^{2}=1-\frac{\varepsilon_{r(\text { training })}}{\sigma^{2}\left[\alpha_{r}\right]}=\frac{\frac{1}{n} \sum_{k=1}^{n}\left(\alpha_{r}\left(\xi^{(k)}\right)-\alpha_{r}^{P C E}\left(\xi^{(k)}\right)\right)^{2}}{\frac{1}{n-1} \sum_{k=1}^{n}\left(\alpha_{r}^{P C E}\left(\xi^{(k)}\right)-\bar{\alpha}_{r}\right)^{2}} ; \quad r=1,2, \ldots
\end{aligned}
$$

where

$$
\bar{\alpha}_{r}=\frac{1}{n} \sum_{k=1}^{n} \alpha_{r}\left(\xi^{(k)}\right)
$$

Taking into consideration the othogonality of the basis functions (discussed previously), the means and variances may be approximated by the truncated PCE as, $\bar{\alpha}_{r} \approx a_{0}$

and

$$
\begin{aligned}
\sigma^{2}\left[\alpha_{r}\right] \approx & \sum_{i_{1}=1}^{\ell}\left(a_{i_{1}}\right)^{-} \operatorname{var}\left(\Gamma_{1}\left(\xi_{i_{1}}\right)\right)+\sum_{i_{1}=1}^{\ell} \sum_{i_{2}=1}\left(a_{i_{1}, i_{2}}\right)^{-} \operatorname{var}\left(\Gamma_{2}\left(\xi_{i_{1}}, \xi_{i_{2}}\right)\right) \\
& +\sum_{i_{1}=1}^{\ell} \sum_{i_{2}=1} \sum_{i_{3}=1}^{i_{2}}\left(a_{i_{1}, i_{2}, i_{3}}\right)^{2} \operatorname{var}\left(\Gamma_{3}\left(\xi_{i_{1}}, \xi_{i_{2}}, \xi_{i_{3}}\right)\right) \\
& +\sum_{i_{1}=1}^{\ell} \sum_{i_{2}=1} \ldots \frac{i_{j-1}}{i_{j}=1}, \ldots, \ldots, \ldots,
\end{aligned}
$$

The $\varepsilon_{\text {training }}$ and $R^{2}$ indices, computed for the wheel test case are shown in Figure 10. In all cases $R^{2}$ values greater than $95.5 \%$ are achieved and the values of $\varepsilon_{\text {training }}$ are seen not to exceed $1.8 \%$. 


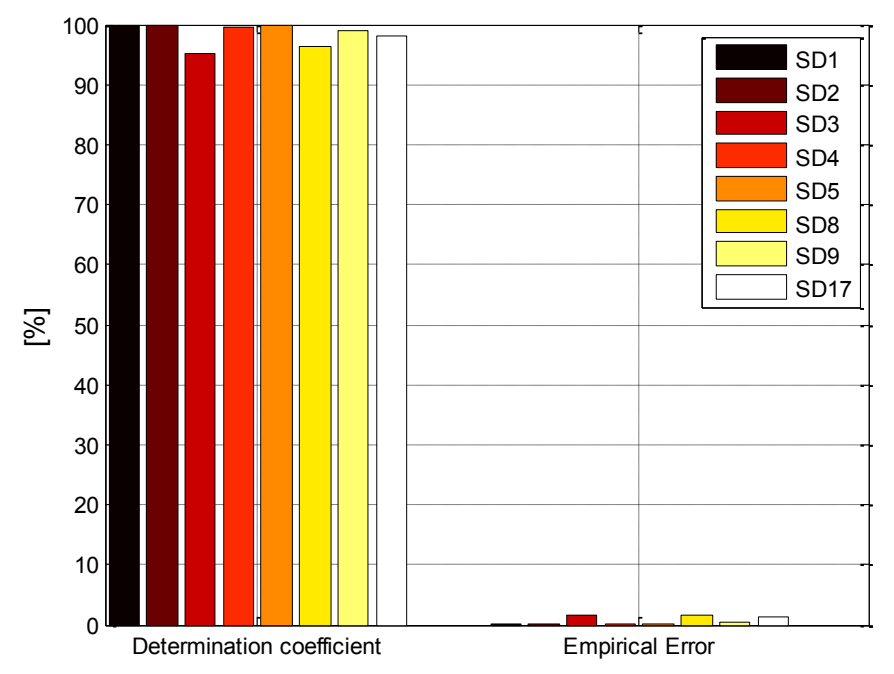

Figure 10: PCE Meta-model accuracy: coefficient $R^{2}$ and $\varepsilon_{(\text {training })}$ for PCE meta-model $(r=4, j=4, p=70, n=200$,$) .$

An evaluation of the variability of the first 20 AGMD was carried out with the inputs sampled according the plan $\mathrm{LHS}_{1}$ previously described. The effect on the AGMD is observed clearly in Figure 11. Certain shape descriptors (AGMD ${ }_{1}$, $\mathrm{AGMD}_{2}, \mathrm{AGMD}_{3}, \mathrm{AGMD}_{5}, \mathrm{AGMD}_{8}, \mathrm{AGMD}_{9}, \mathrm{AGMD}_{17}$ ) are clearly the ones most greatly influenced by a change of the input parameters. Only those with both significant mean values and variance are considered in the analysis. In the box plot the central red mark corresponds to the median, the edges of the box define the $25^{\text {th }}$ and $75^{\text {th }}$ percentiles while the whiskers extend to $99.3 \%$ of the output data.

The local sensitivity of the stress field to a small change in any one of the four input parameters (in the sense of the derivative at a point in the parameter space) is virtually identical to the sensitivity of the other three, except for changes in sign. Thus, the variability of AGMD with $I_{\text {fit }}$ shown in Figure 11 is sufficient for a complete understanding of the incremental variation in the stress field with any one of the four inputs.

The inputs may be combined in the form of a single input, the interference fit, defined as, 
$I_{f i t}=\left[\begin{array}{llll}+1 & -1 & +1 & +1\end{array}\right]\left\{\begin{array}{c}\phi_{d} \\ \phi_{t} \\ t_{d} \\ t_{r}\end{array}\right\}$

Deviations from circularity were not considered, since this would complicate the analysis unnecessarily, without adding to the understanding of the methodology.
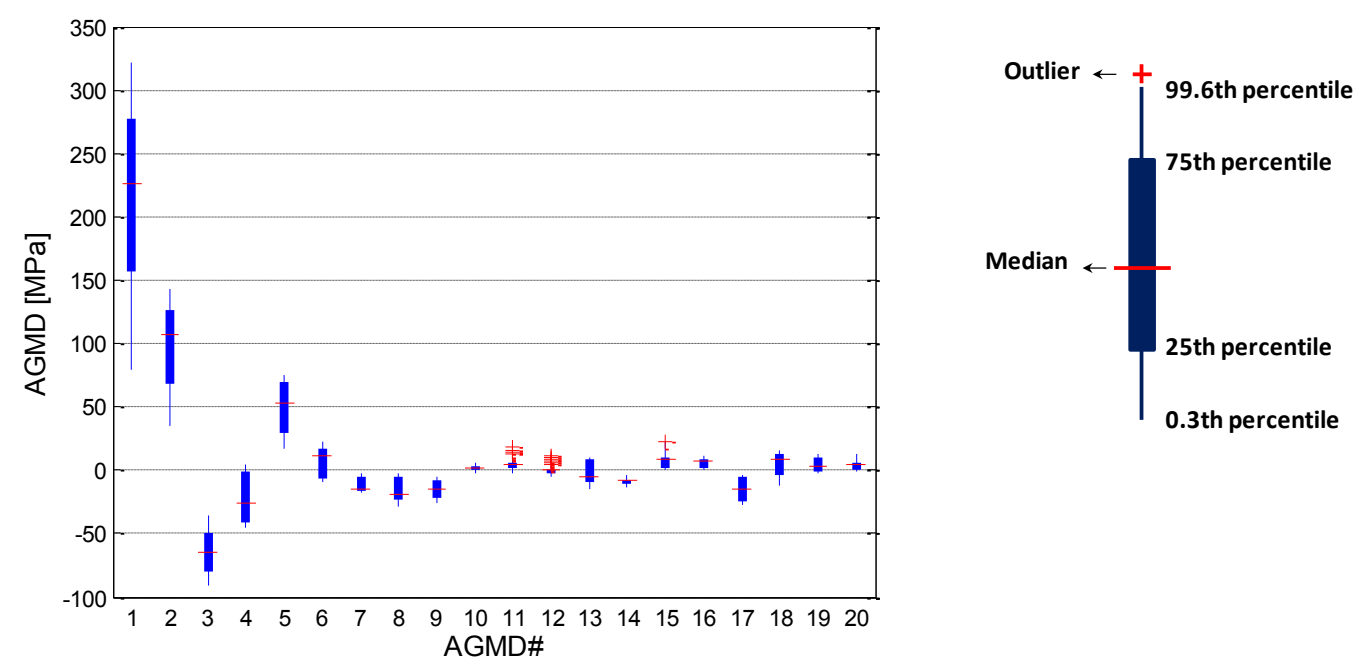

Figure 11: AGMD variability

It is seen from Figure 12 that the input-output relationship is highly nonlinear. The individual input parameters, sampled according to $\mathrm{LHS}_{2}, \mathrm{LHS}_{3}, \mathrm{LHS}_{4}$ and $\mathrm{LHS}_{5}$, contribute to the interference fit in certain ranges. For example the pink sample points, denoting the rim thickness, appear in the central region of the interference fit between approximately 1 and $1.2 \mathrm{~mm}$ of interference. On the other hand the dark blue points that denote rim diameter variability seem to be spread over almost the complete range of interference. This is because of the manufacturing tolerances placed on the different inputs as indicated by the 'range' in Table 1. The open-circle sample points correspond to sample plan $\mathrm{LHS}_{1}$ where all the inputs are sampled together. Sampling plans $\mathrm{LHS}_{2}, \mathrm{LHS}_{3}, \mathrm{LHS}_{4}, \mathrm{LHS}_{5}$ neglect any interaction effects between the inputs. In general it is possible to establish a direct link between the input uncertainty and the variability of the output response, to be quantified in the following section. 

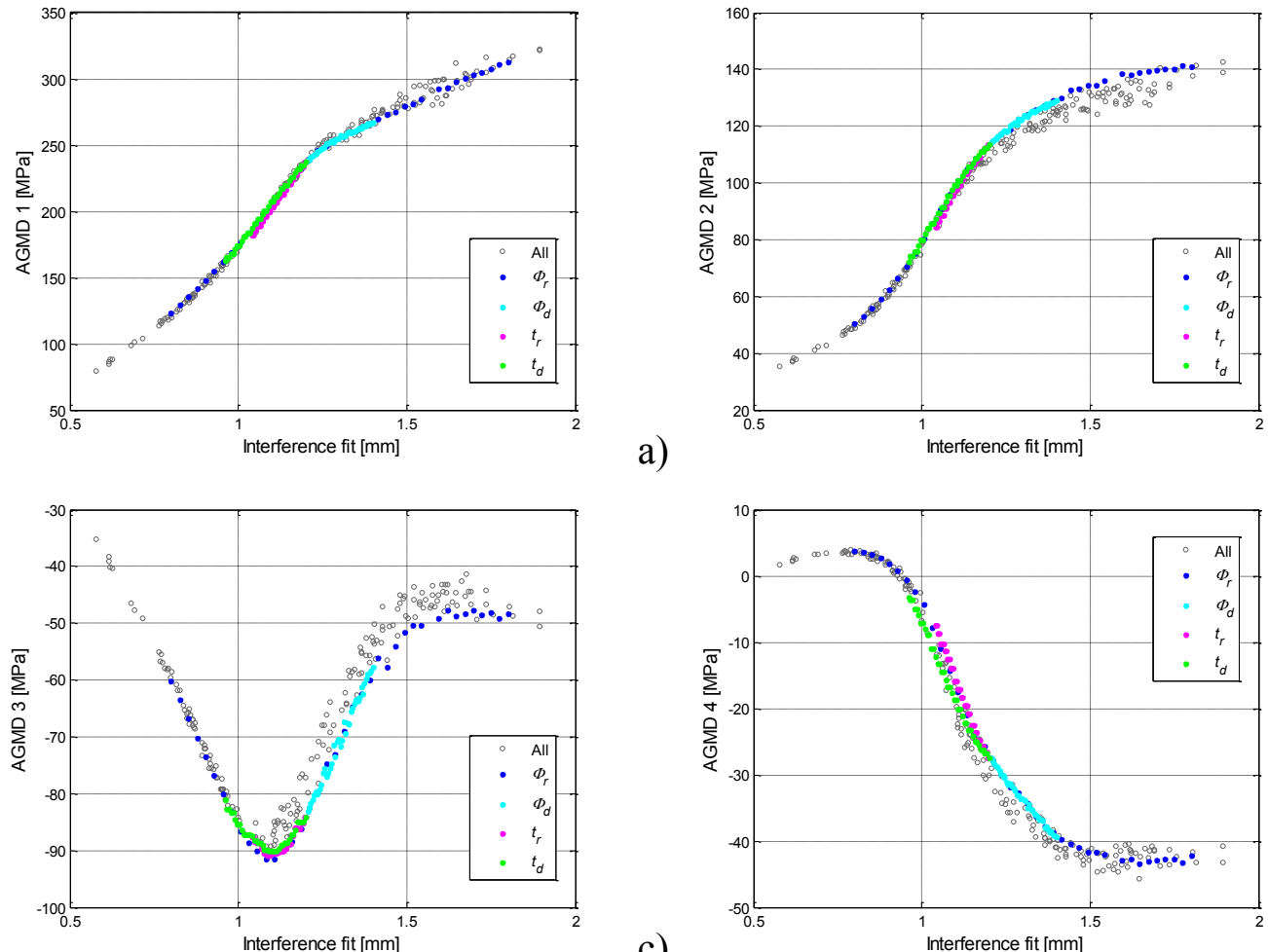

c)
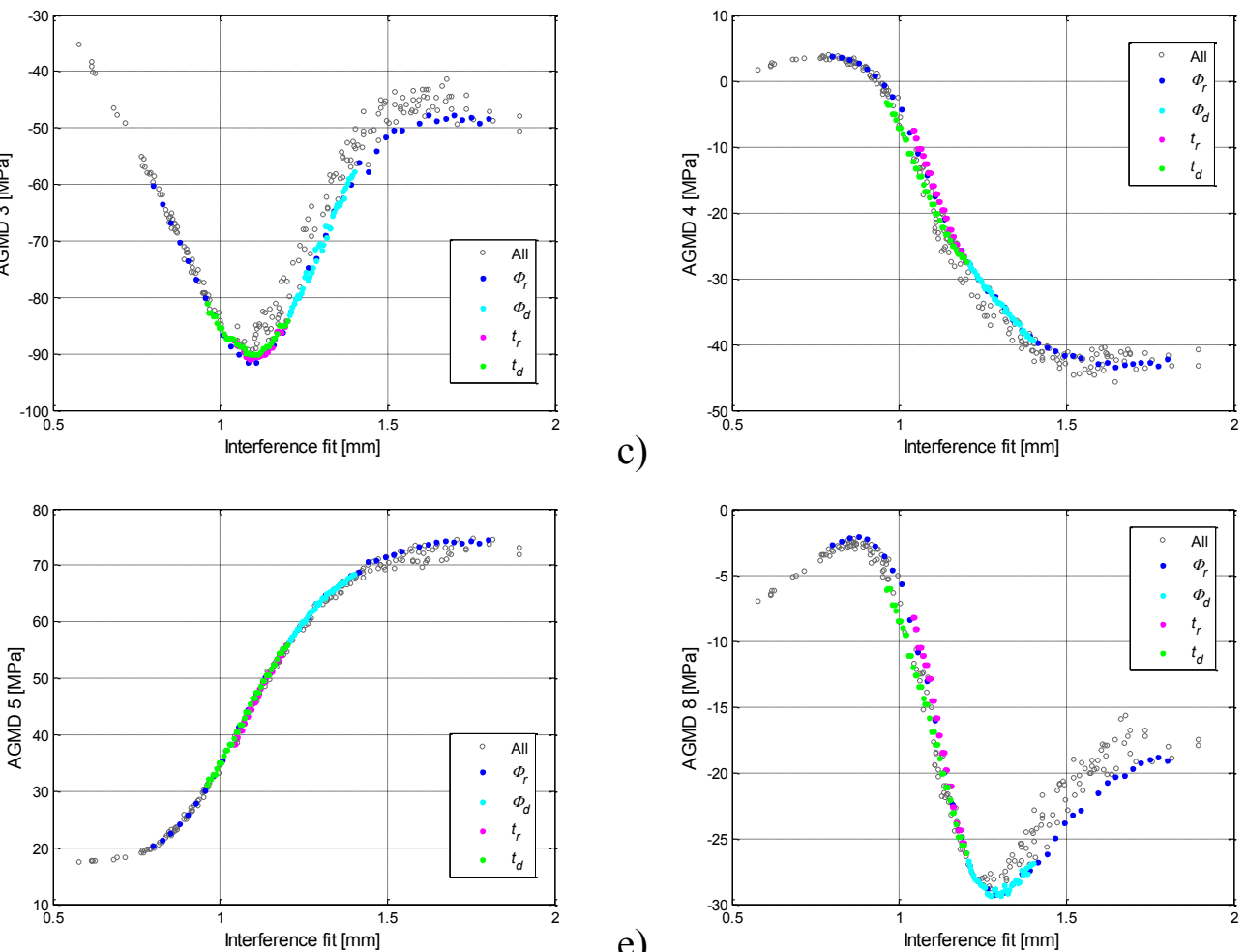

e)

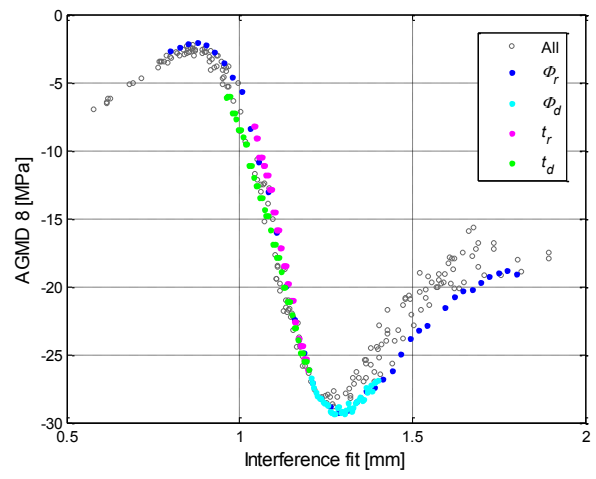

d)
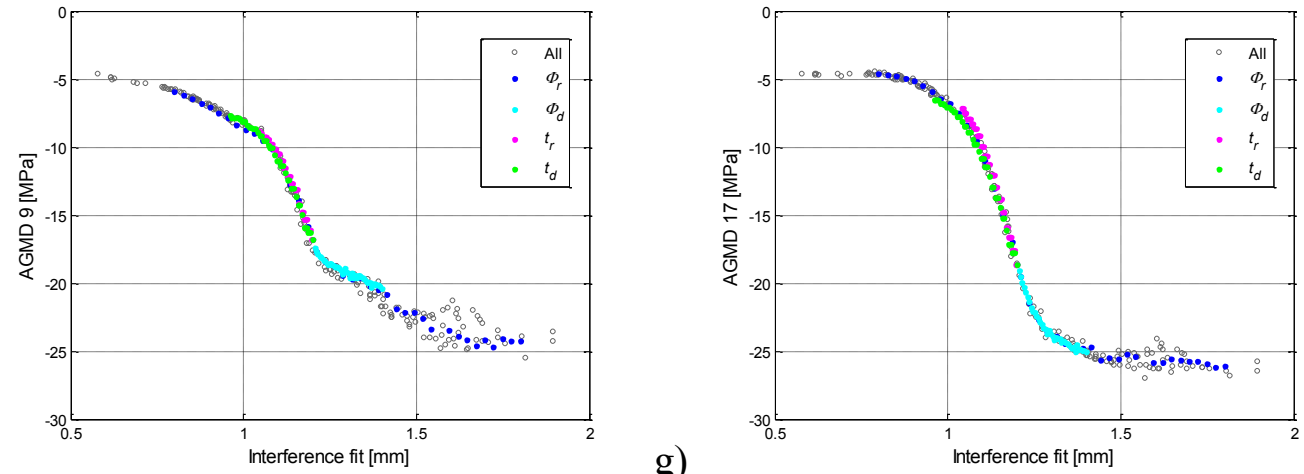

g)

Figure 12: Adaptive geometric moment descriptors (region 1): a) $\mathrm{AGMD}_{1}, \mathrm{~b}$ ) h) $\mathrm{AGMD}_{2}$, c) $\mathrm{AGMD}_{3}$, d) AGMD 4 , e) AGMD 5 , f) AGMD 8 ,g) AGMD 9 ,h) AGMD 17 . 


\section{Global sensitivity analysis}

Unlike local sensitivity, the global sensitivity (GS) indices include the whole range of input uncertainty and provide an estimate of the fractional contribution of each input to the variance of the output. As well as the direct effect of a particular uncertain input, the GS includes the sum of all the contributions arising from combination with other inputs and may be used to assess the potential for tolerance reduction within an existing design. By controlling the most dominant inputs it should be possible to reduce the output variability.

The global sensitivities, expressed using the Sobol' indices ${ }^{14}$, are the contribution of the $t^{\text {th }}$ input to the total variance of the output. This partial variance may be written as,

$$
\begin{aligned}
& \sigma_{t}^{2}\left[\alpha_{r}\right] \approx\left(a_{i_{t}}\right)^{2} \operatorname{var}\left(\Gamma_{1}\left(\xi_{i_{t}}\right)\right)+\sum_{i_{1}=1}^{\ell}\left(a_{i_{1}, i_{t}}\right)^{2} \operatorname{var}\left(\Gamma_{2}\left(\xi_{i_{1}}, \xi_{i_{t}}\right)\right) \\
& +\sum_{i_{1}=1}^{\ell} \sum_{i_{2}=1}\left(a_{i_{1}, i_{2}, i_{t}}\right)^{2} \operatorname{var}\left(\Gamma_{3}\left(\xi_{i_{1}}, \xi_{i_{2}}, \xi_{i_{t}}\right)\right)
\end{aligned}
$$

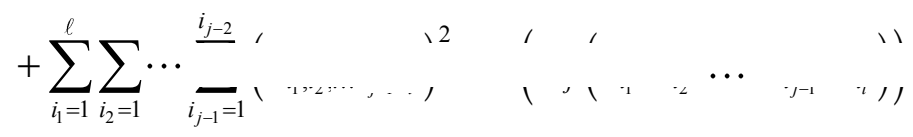

where $i_{t} \in\left\{i_{\kappa}\right\}_{\kappa=1}^{j-1}$. It is seen that those inputs that do not combine with $t$ in equations (4) and (11) are excluded from (13). The first-order global sensitivities of each output with respect to each input are then given by bringing together all the terms in equation (13) that are polynomial functions of $\xi_{i_{t}}$ alone,

$$
\begin{gathered}
S_{r, t}^{1}=\left(\left(a_{i_{t}}\right)^{2} \operatorname{var}\left(\Gamma_{1}\left(\xi_{i_{t}}\right)\right)+\left(a_{i_{t}, i_{t}}\right)^{2} \operatorname{var}\left(\Gamma_{2}\left(\xi_{i_{t}}, \xi_{i_{t}}\right)\right)\right. \\
\left.+\left(a_{i_{t}, i_{t}, i_{t}}\right)^{2} \operatorname{var}\left(\Gamma_{3}\left(\xi_{i_{t}}, \xi_{i_{t}}, \xi_{i_{t}}\right)\right)+\ldots\right) / \frac{1}{n-1} \sum_{k=1}^{n}\left(\alpha_{r}\left(\xi^{(k)}\right)-\bar{\alpha}_{r}\right)^{2} \\
r=1,2, \ldots
\end{gathered}
$$

The second order sensitivities are given by the combined contributions of all the terms in equation (13) that are multivariate polynomial functions in $\xi_{i_{t}}$ and one other input $\xi_{i_{1}}$, 


$$
\begin{gathered}
S_{r, t}^{2}=\left(\sum_{\substack{i_{i}=1 \\
i_{1} \neq i_{t}}}^{\ell}\left(a_{i_{1}, i_{t}}\right)^{-} \operatorname{var}\left(\Gamma_{2}\left(\xi_{i_{1}}, \xi_{i_{t}}\right)\right)+\sum_{\substack{i_{1}=1 \\
i_{1} \neq i_{t}}}^{\ell}\left(a_{i_{1}, i_{1}, i_{t}}\right)^{-} \operatorname{var}\left(\Gamma_{3}\left(\xi_{i_{1}}, \xi_{i_{1}}, \xi_{i_{t}}\right)\right)\right. \\
\left.+\sum_{\substack{i_{1}=1 \\
i_{1} \neq i_{t}}}^{\ell}\left(a_{i_{1}, i_{t}, i_{t}}\right)^{2} \operatorname{var}\left(\Gamma_{3}\left(\xi_{i_{1}}, \xi_{i_{t}}, \xi_{i_{t}}\right)\right)+\ldots\right) / \frac{1}{n-1} \sum_{k=1}^{n}\left(\alpha_{r}\left(\xi^{(k)}\right)-\bar{\alpha}_{r}\right)^{2} \\
r=1,2, \ldots
\end{gathered}
$$

The third and higher order sensitivities follow logically from equations (14) and (15).

The first-order results, in Figure 13(a), show strong sensitivity of each of the significant AGMDs to uncertainty in the rim diameter. This is to be expected since the tolerance range on $\phi_{\text {rim }}$ is much greater than the tolerances on the other inputs. The combination effects are seen from Figure 13(b) to be small, so that polynomial terms involving the products of inputs $\xi_{i}$ contribute only slightly to the second order sensitivity. The total sensitivity is given in Figure 13(c) as the sum of the contributions in Figure 13(a) and 13(b) together with higher order terms. The eight AGMDs are found to be quite uniform in their sensitivity to the inputs.

The global sensitivity analysis generally provides a more thorough assessment than the screening method ${ }^{15,16}$ which offers a simple and rapid approach to perform a first sensitivity analysis. The latter does not allow investigation of input interactions, but gives a rough idea on how each input factor can affect the quantity of interest. It does however agree well with the global sensitivity values in the present case because of the small second-order terms ${ }^{17}$. 


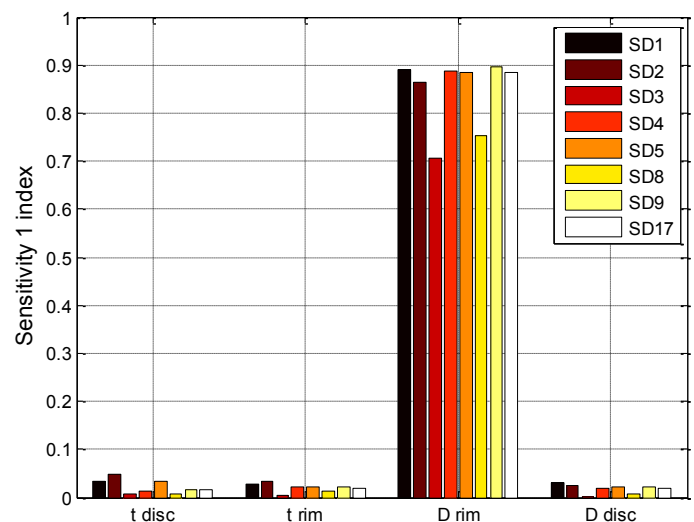

a)

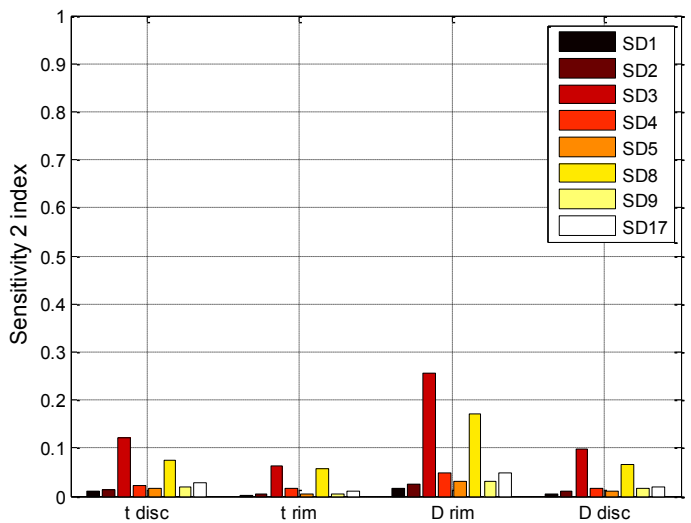

b)

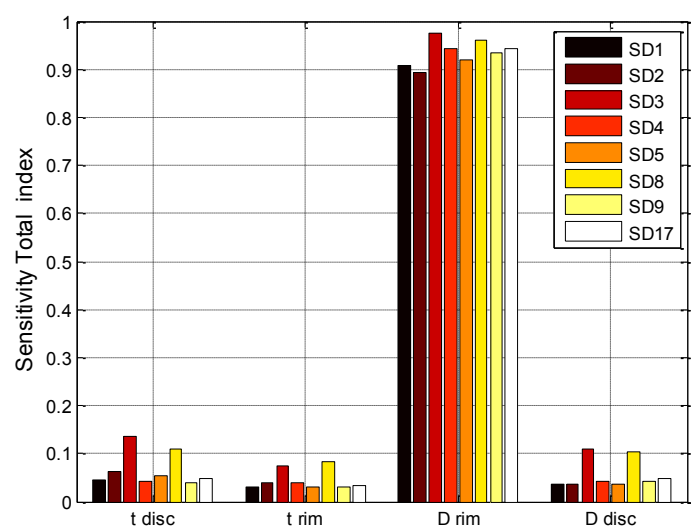

c)

Figure 13: Global sensitivity indices: a) $1^{\text {st }}$ order sensitivity $\left(\mathrm{LHS}_{1}\right)$, b) $2^{\text {nd }}$ order sensitivity $\left.\left(\mathrm{LHS}_{1}\right), \mathrm{c}\right)$ Total sensitivity $\left(\mathrm{LHS}_{1}\right)$ 


\section{Tolerances reduction for stochastic model design}

In this section a revised sample plan, shown in Table 2, will be considered. It is seen by comparison with Table 1 that $\phi_{r}$ is now much more tightly constrained and that a new input, the Young modulus $E_{l}$ of the material, has been added. Its variability is chosen to be bounded between $204 \mathrm{GPa}$ and $216 \mathrm{GPa}$, i.e. $\pm 3 \%$ uncertainty.

Table 2. Sampling plans $\left(\mathrm{LHS}_{6}\right)$ considering tolerance reduction on $\phi_{r}$ and material factor $\mathrm{E}_{1}$ in addition

\begin{tabular}{lccccc}
\hline \multirow{2}{*}{ Input } & \multicolumn{2}{c}{ LHS $_{\mathbf{6}}(\mathrm{n}=200)$} & & Total Sensitivity \\
\cline { 2 - 3 } \cline { 6 - 6 } & $\begin{array}{c}\text { Lower } \\
\text { bound }\end{array}$ & $\begin{array}{r}\text { Upper } \\
\text { bound }\end{array}$ & Range & & $\begin{array}{c}\text { AGMD-based } \\
\text { ranking }\end{array}$ \\
\hline Disc diameter $\phi_{d}[\mathrm{~mm}]$ & 359.8 & 360.0 & 0.2 & & 2 \\
Rim diameter $\phi_{r}[\mathrm{~mm}]$ & 366.4 & 366.6 & 0.2 & & 3 \\
Disc thickness $t_{d}[\mathrm{~mm}]$ & 4.56 & 4.80 & 0.24 & \\
Rim thickness $t_{r}[\mathrm{~mm}]$ & 3.04 & 3.20 & 0.16 & \\
Young's modulus $E_{1}[\mathrm{GPa}]$ & 204 & 216 & 12 & & \\
\hline
\end{tabular}

The sensitivity of each shape descriptor shown in Figure 14 reveals a new rankingbased parameter classification with the result that the variability of the first 20 AGMDs is very clearly tightened, as demonstrated in Figure 15.

The variability of the first three AGMD with tolerance on $\phi_{r}$ are shown in Figure 16. As seen in Figure 17 (a) the $75^{\text {th }}$ percentile of $A G M D_{1}$, the most significant shape descriptor, shows a reduction of around $100 \mathrm{MPa}$ when the tolerance is reduced from $1 \mathrm{~mm}$ to $0.2 \mathrm{~mm}$. Significant reductions are also seen in $\mathrm{AGMD}_{2}$ and $\mathrm{AGMD}_{3}$.

By analysing the work done by the force acting on the disc during the fitting process, rather than the AGMD of the full field data at the end of the fitting process, similar conclusions can be obtained. Again the input $\phi_{r}$ is seen to be mainly responsible for the variability of the output $\left(\mathrm{LHS}_{1}\right)$. When the tolerance range is changed (as in LHS$_{6}$ ), then the disc thickness becomes more relevant as shown in Figure 14, and the other three inputs $\left(\phi_{d} t_{d} t_{r}\right)$ contribute in a similar way to the total output variance. The added input $E_{1}$ has very little effect on the variability of the AGMDs. The result is a robust design methodology, testable during the production, for 
investigation the effect of manufacturing tolerances on the performance of engineering components.

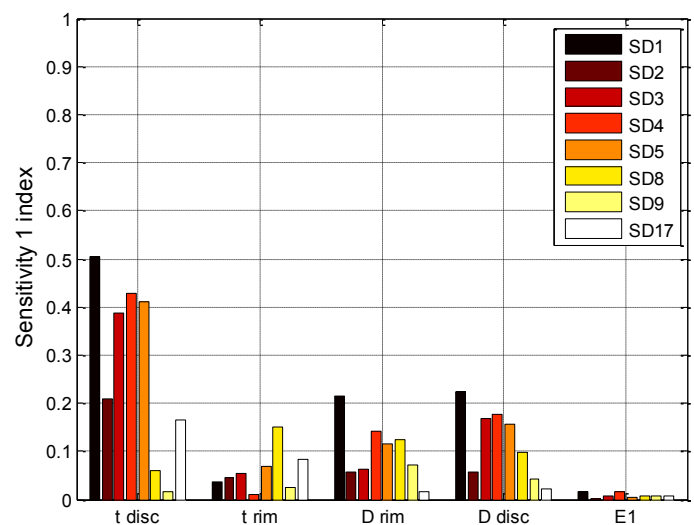

a)

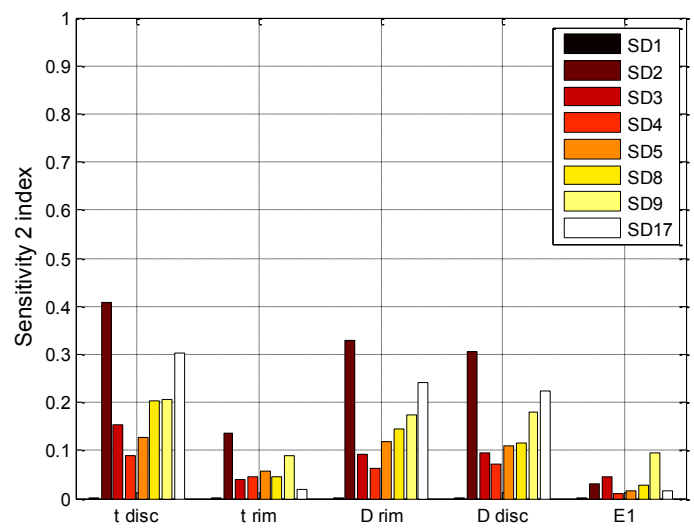

b)

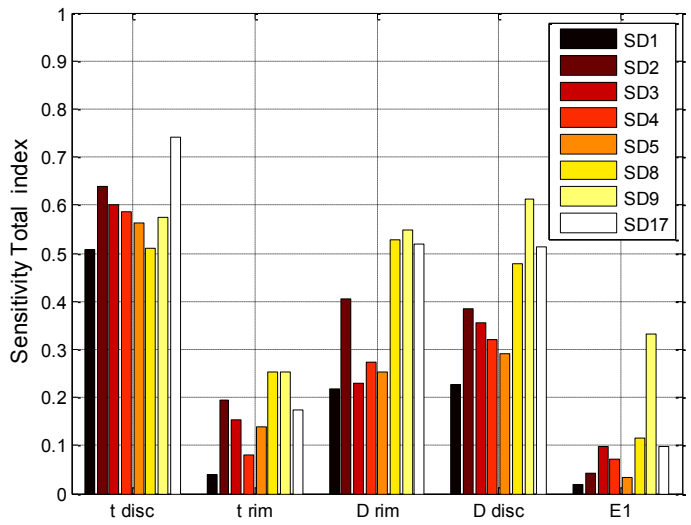

c)

Figure 14: Global sensitivity indices: a) $1^{\text {st }}$ order sensitivity $\left(\mathrm{LHS}_{6}\right)$, b) $2^{\text {nd }}$ order sensitivity $\left(\mathrm{LHS}_{6}\right)$, c) Total sensitivity $\left(\mathrm{LHS}_{6}\right)$ 


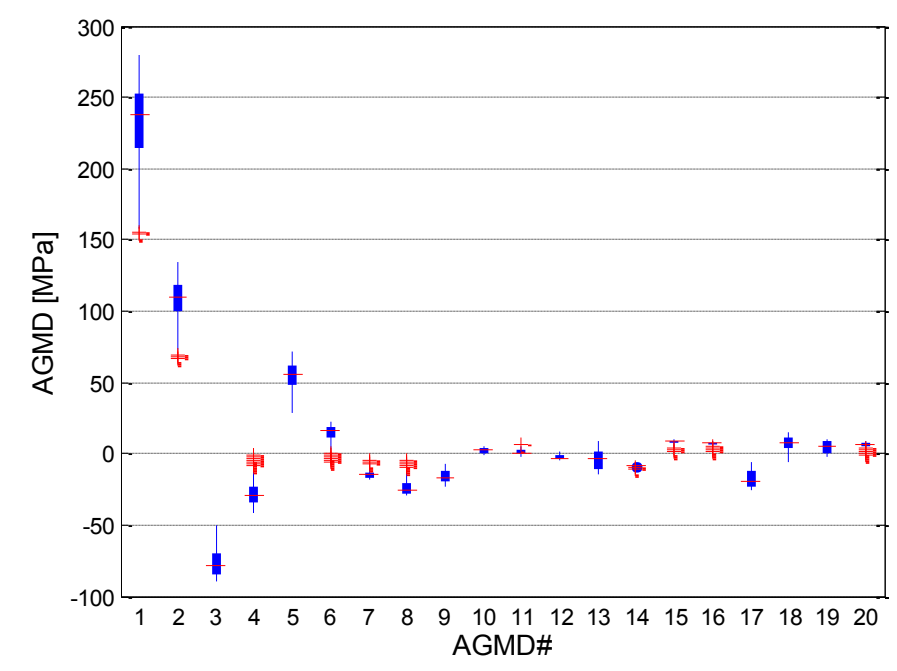

Figure 15: AGMD variability

\section{Conclusions}

A methodology is developed for the design of engineering components that are assembled by means of an interference fit. Manufacturing tolerances lead to variability in the stress field of the mating parts and it is important be able to understand the influence of each of several tolerances individually. This is achieved using a combination of image decomposition of the stress field to provide a small number of output shape descriptors, the polynomial chaos expansion to provide a probabilistic mapping from the input tolerances and the output shape descriptors, and global sensitivity analysis which shows the extent to which each input affects the observed variance of every output. This provides a design tool for the setting of manufacturing tolerances in order to achieve a benign stress field and desirable performance of the assembled structure. The methodology is illustrated using the practical example of a press-fitted car wheel. 


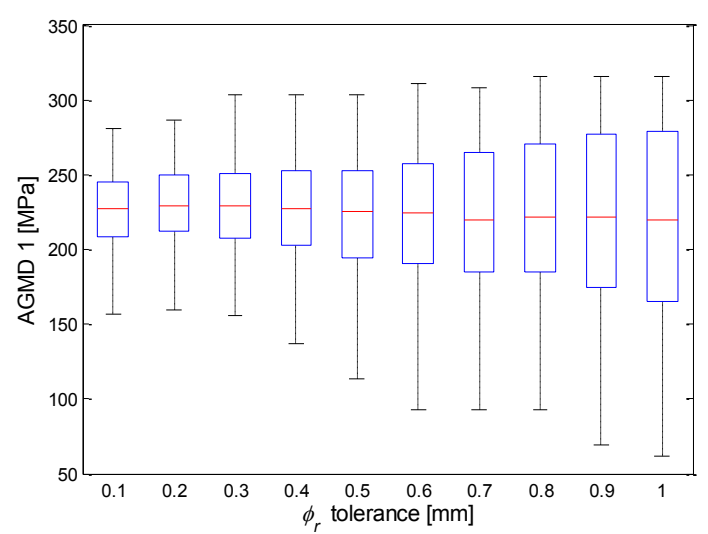

(a)

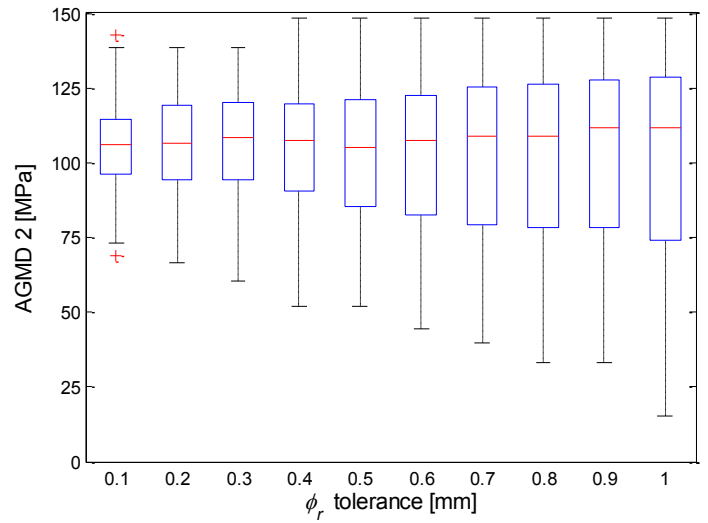

(b)

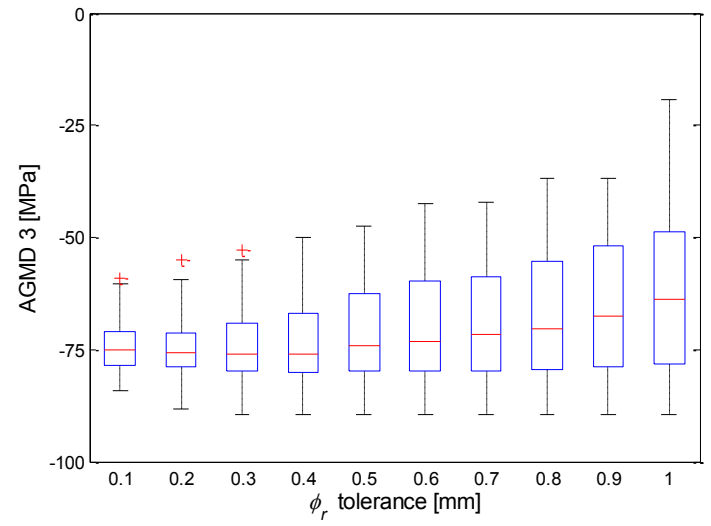

(c)

Figure 16: Variability in the first 3 AGMD with loosening of tolerance 


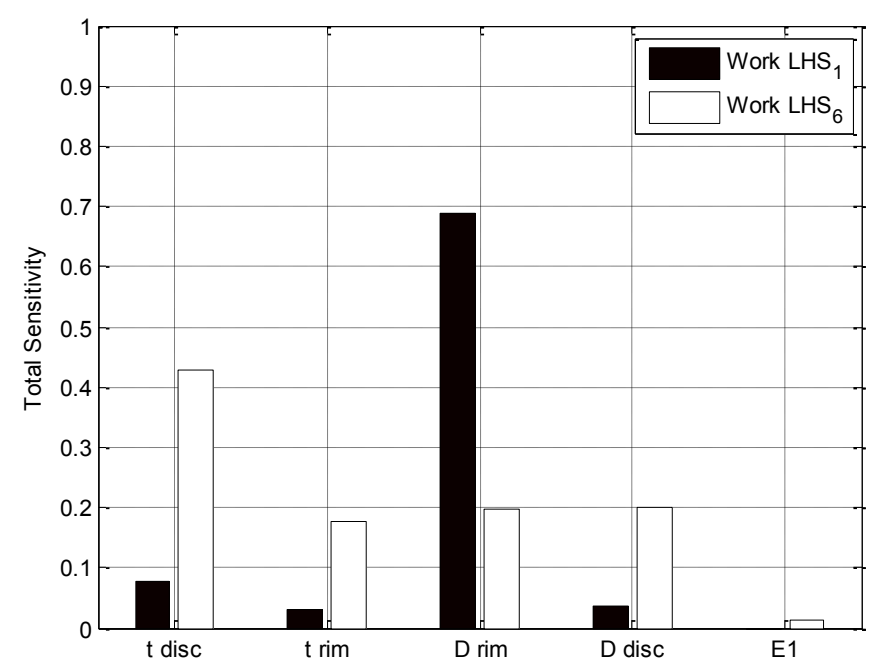

Figure 17: Total sensitivity indices of Work $W$ for two sampling plan: $\mathrm{LHS}_{1}$ and LHS $_{6}$ (PCE meta-model: $\mathrm{R}^{2}{ }_{\text {LHS1 }}=0.998, \mathrm{R}^{2}{ }_{\text {LHS1 }}=0.999$ )

\section{References}

1. Wang X, Zhang X. Simulation of dynamic cornering fatigue test of a steel passenger car wheel. Int J Fatigue [Internet]. 2010 Feb [cited $2013 \mathrm{Nov}$ 22];32(2):434-442. Available from:

http://linkinghub.elsevier.com/retrieve/pii/S0142112309002722

2. Grubisic V, Fischer G. Procedure for optimal lightweight design and durability testing of wheels. Int J Veh Des. 1984;5(6):659-671.

3. Wang W, Mottershead JE, Sebastian CM, Patterson E a. Shape features and finite element model updating from full-field strain data. Int J Solids Struct [Internet]. 2011 Jun [cited 2013 Nov 22];48(11-12):1644-1657. Available from: http://linkinghub.elsevier.com/retrieve/pii/S0020768311000692

4. Wang W, Mottershead JE, Siebert T, Pipino A. Frequency response functions of shape features from full-field vibration measurements using digital image correlation. Mech Syst Signal Process [Internet]. 2012;28:333-347. Available from: http://dx.doi.org/10.1016/j.ymssp.2011.11.023

5. Ming-Kuei H. Visual pattern recognition by moment invariants. IRE Trans Inf Theory. 1962;:66-70.

6. Teague MR. Image analysis via the general theory of momentst. J Opt Soc Am. 1980;70(8). 
7. Wang W, Mottershead JE. Adaptive moment descriptors for full-field strain and displacement measurements. J Strain Anal Eng Des [Internet]. 2012 Oct 22 [cited 2013 Nov 22];48(1):16-35. Available from: http://sdj.sagepub.com/lookup/doi/10.1177/0309324712460485

8. Golub G, Van Loan C. Matrix Computations. Baltimore: Johns Hopkins University Press; 1996.

9. Aurenhammer F. Voronoi Diagrams - A Survey of a Fundamental Data Structure. ACM Comput Surv [Internet]. 1991;23(3):345-405. Available from: http://portal.acm.org/citation.cfm?doid=116873.116880

10. Devore JL. Probability and Statistics for engineering and the Sciences. Brooks/Cole, Boston (USA); 2012.

11. Johnson ME, L.M. M, Ylvisaker D. Minimax and maximin distance designs. J Stat Plan Inference. 1990;26:131-148.

12. Ghanem RG, Spanos PD. Stochastic finite elements - A spectral approach. Springer Verlag; 2003.

13. Sudret B, Blatman G. Efficient computation of global sensitivity indices using sparse polynomial chaos expansions. Reliab Eng Syst Saf [Internet]. 2010 Nov [cited 2013 Nov 6];95(11):1216-1229. Available from: http://linkinghub.elsevier.com/retrieve/pii/S0951832010001493

14. Sobol IM. Global sensitivity indices for nonlinear mathematical models and their Monte Carlo estimates. Math Comput Simul. 2001;55:271-280.

15. Saltelli A, Ratto M, Andres T, Campolongo F, Cariboni J, Gatelli D, et al. Global sensitivity analyses: The Primer. New York: John Wiley and Sons; 2008 .

16. Forrester AIJ, Sobester A, Keane A. Engineering Design via Surrogate Modelling. 2008.

17. Marcuccio G, Mottershead JE, Bonisoli E, Tornincasa S. Geometrical and Dimensional Uncertainties Effects Quantification for Stress / Strain Field Characterization. In: Prognostic and System Health Management. 2013. p. 1099-1104. 